\title{
Fractional flow reserve guided percutaneous coronary intervention optimization directed by high-definition intravascular ultrasound versus standard of care: Rationale and study design of the prospective randomized FFR- REACT trial
}

Laurens J. C. van Zandvoort, BSc, Kaneshka Masdjedi, MD, Maria Natalia Tovar Forero, MD, Mattie J. Lenzen, PhD, Jurgen Ligthart, RT, Roberto Diletti, MD, PhD, Miguel E. Lemmert, MD, PhD, Jeroen Wilschut, MD, Peter P. T. de Jaegere, MD, PhD, Felix Zijlstra, MD, PhD, Nicolas M. van Mieghem, MD, PhD, and Joost Daemen, MD, PhD Thoraxcenter, Erasmus Medical Center

Background Post percutaneous coronary intervention (PCI) fractional flow reserve (FFR) is a significant predictor of major adverse cardiac events (MACE). The rationale for low post procedural FFR values often remains elusive based on angiographic findings alone, warranting further assessment using an FFR pullback or additional intravascular imaging. It is currently unknown if additional interventions intended to improve the $\mathrm{PCl}$, decrease MACE rates.

Study design The FFR REACT trial is a prospective, single-center randomized controlled trial in which 290 patients with a post PCI FFR $<0.90$ will be randomized (1:1) to either standard of care (no additional intervention) or intravascular ultrasound (IVUS)-directed optimization of the FFR (treatment arm). Eligible patients are those treated with angiographically successful PCI for (un)stable angina or non-ST elevation myocardial infarction (MI). Assuming $45 \%$ of patients will have a post $\mathrm{PCl}$ FFR $<0.90$, approximately 640 patients undergoing PCl will need to be enrolled. Patients with a post $\mathrm{PCl} F F R \geq 0.90$ will be enrolled in a prospective registry. The primary end point is defined as a composite of cardiac death, target vessel $\mathrm{Ml}$ and clinically driven target vessel revascularisation (target vessel failure) at 1 year. Secondary end points will consist of individual components of the primary end point, procedural success, stent thrombosis and correlations on clinical outcome, changes in post $\mathrm{PCl} \mathrm{Pd} / \mathrm{Pa}$ and FFR and IVUS derived dimensions. All patients will be followed for 3 years.

Conclusion The FFR-REACT trial is designed to explore the potential benefit of HD-IVUS-guided PCl optimization in patients with a post PCI FFR $<0.90$ (Dutch trial register: NTR671 1). (Am Heart J 2019;213:66-72.)

\section{Background}

Accurate angiographic assessment of the severity and hemodynamic importance of coronary artery stenosis can be challenging and proved to be frequently unreliable. ${ }^{1,2}$ Previous studies demonstrated that routine pre-

From the and Department of cardiology, Thoraxcenter, Erasmus Medical Center. RCT\# NTR6711.

Submitted December 3, 2018; accepted March 27, 2019.

Reprint requests: Dr. Joost Daemen, MD, PhD, Department of Cardiology, Room Rg-628, Erasmus University Medical Center, P.O. Box 2040, 3000, CA, Rotterdam, The

Netherlands.

E-mail: j.daemen@erasmusmc.nl

0002-8703

(c) 2019 Elsevier Inc. All rights reserved.

https://doi.org/10.1016/i.ahj.2019.03.017 procedural fractional flow reserve (FFR) in patients with multivessel coronary artery disease undergoing percutaneous coronary intervention (PCI) with drugeluting stents significantly reduces the rate of the composite end point of death, nonfatal myocardial infarction (MI), and repeat revascularization at 1 year as compared to angiographic guided PCI. ${ }^{3}$ More recently, FFR after stenting proved to be a strong and independent predictor of major adverse cardiac events (MACE) at 1 year. ${ }^{4} \mathrm{~A}$ contemporary meta-analysis on the clinical impact of post PCI FFR values showed that an FFR $<0.90$ is associated with an increased risk of target vessel revascularization (TVR). ${ }^{5}$

A number of factors might cause a post-PCI pressure drop over a treated segment including residual disease in the proximal or distal segment, a geographically 
misplaced stent, stent underexpansion, malapposition, plaque protrusion, edge dissection and plaque shift. ${ }^{6,7}$ While these findings are not always readily apparent on coronary angiography alone, high definition (HD) intravascular ultrasound (IVUS) demonstrated to be a powerful tool to detect potential causes for low FFR post stenting. More specifically, these issues proved to be more frequently present in patients with low as compared to high post PCI FFR. ${ }^{7}$ The latter adds to the substantial body of evidence on the benefit of IVUSguided PCI as compared to angiography-guided PCI in improving long-term outcomes. ${ }^{8,9}$

While post PCI FFR is at present only rarely performed in routine clinical practice, an FFR after stenting $<0.90$ proved to be present in approximately $45 \%$ of the patients. ${ }^{10}$ Additionally, IVUS was able to detect problems of intraluminal obstruction in up to $84 \%$ of those cases. ${ }^{7}$ It is currently unknown if additional interventions with the intent to optimize post procedure FFR improve patient outcome.

The rationale and design of the FFR REACT trial was based on a simple and fast way of measuring post PCI FFR using a small microcatheter over the previously used coronary guidewire. Although a substantial body of evidence exists towards a pressure wire based post PCI FFR of 0.90 to predict MACE, at the moment no clear cutoff for post PCI FFR value as measured with a microcatheter to predict events has been established. ${ }^{4,5}$ The potential findings and clinical implications of this study might open the door to a more frequent use of post PCI physiological assessment with the intention to further reduce the risk of future MACE with the help of IVUS.

\section{Study aims}

To assess if FFR guided PCI optimization directed by HD-IVUS in patients with an increased risk for MACE (post-PCI FFR below 0.90) will improve clinical outcome and reduce target vessel failure, a composite of cardiac death, target-vessel myocardial infarction and clinically driven TVR at 1 year.

\section{Study design and methods}

The FFR REACT trial is a prospective, investigator initiated single-center randomized controlled trial in which 290 patients with a post PCI FFR $<0.90$ will be randomized $(1: 1)$ to either standard of care (no additional intervention, control arm) or IVUS-directed optimization of the FFR (treatment arm). Eligible patients are those treated with angiographically successful PCI for stable or unstable angina or a non-ST elevation myocardial infarction (MI). Patients with a post PCI FFR $\geq 0.90$ will be enrolled in a prospective registry. All patients will be included in the Erasmus Medical Center (MC), the Netherlands, and followed for up to 3 years after PCI.
The study flowchart is depicted in Figure 1. The study protocol was approved by our local ethical committee on the 26th of October 2017 (MEC-2017-489). Financial support is provided by ACIST Medical Systems, Inc.. The Erasmus Medical Center is totally independent from ACIST Medical Systems, Inc. regarding the conduct of the study and the medical treatment of patients and study subjects. The authors are solely responsible for the design and conduct of this study, all study analyses, the drafting and editing of the paper and its final contents. The study is in accordance with Good Clinical Practices (GCP), ISO14155 and with the Declaration of Helsinki (64th WMA General Assembly, Fortaleza, Brazil, October 2013). The study is registered at the Dutch trial register: NTR6711.

\section{Study population}

With the assumption that post PCI FFR will be $<0.90$ in $45 \%$ of the patients, an estimated number of 640 patients will be enrolled in order to be able to randomize 290 patients. Detailed in- and exclusion criteria are depicted in Table I. Each patient must sign and date the approved informed consent form after the study has been thoroughly explained.

Study endpoints. The primary endpoint will be assessed at 1-year follow-up and is defined as target vessel failure, a composite of cardiac death, target vessel Q-wave or non-Q wave MI and clinically driven TVR.

Secondary endpoints consist of the individual components of the primary endpoint at 6 months, 1, 2 and 3 years, along with other clinical endpoints: all-cause death, any coronary revascularization, non-fatal MI, stent thrombosis (according the ARC criteria ${ }^{11}$ ), stroke, periprocedural complications and acute kidney injury. Procedural characteristics such as contrast medium usage, number of stents, total stent length and procedural time will be compared between groups. Additionally, correlations between changes in post procedural FFR and Pd/Pa and luminal dimensions on IVUS due to potential optimization will be assessed. Table II depicts both primary and secondary endpoints.

Finally, operator PCI strategy will be assessed at multiple time points.

\section{Blinding and randomization}

Patient randomization will be initiated through a webbased application (ALEA, Formvision, Utrecht, The Netherlands). In order to prevent a disturbed allocation between treatment arms, a block randomization will be used, varying between four and six in size. Subjects will be blinded to the post procedural FFR and subsequent treatment allocation. In order to monitor the level of blinding, the perceived treatment allocation will be inquired at the 1 year clinical follow-up visit. Furthermore, the procedure report will not contain any details 


\section{Figure 1}

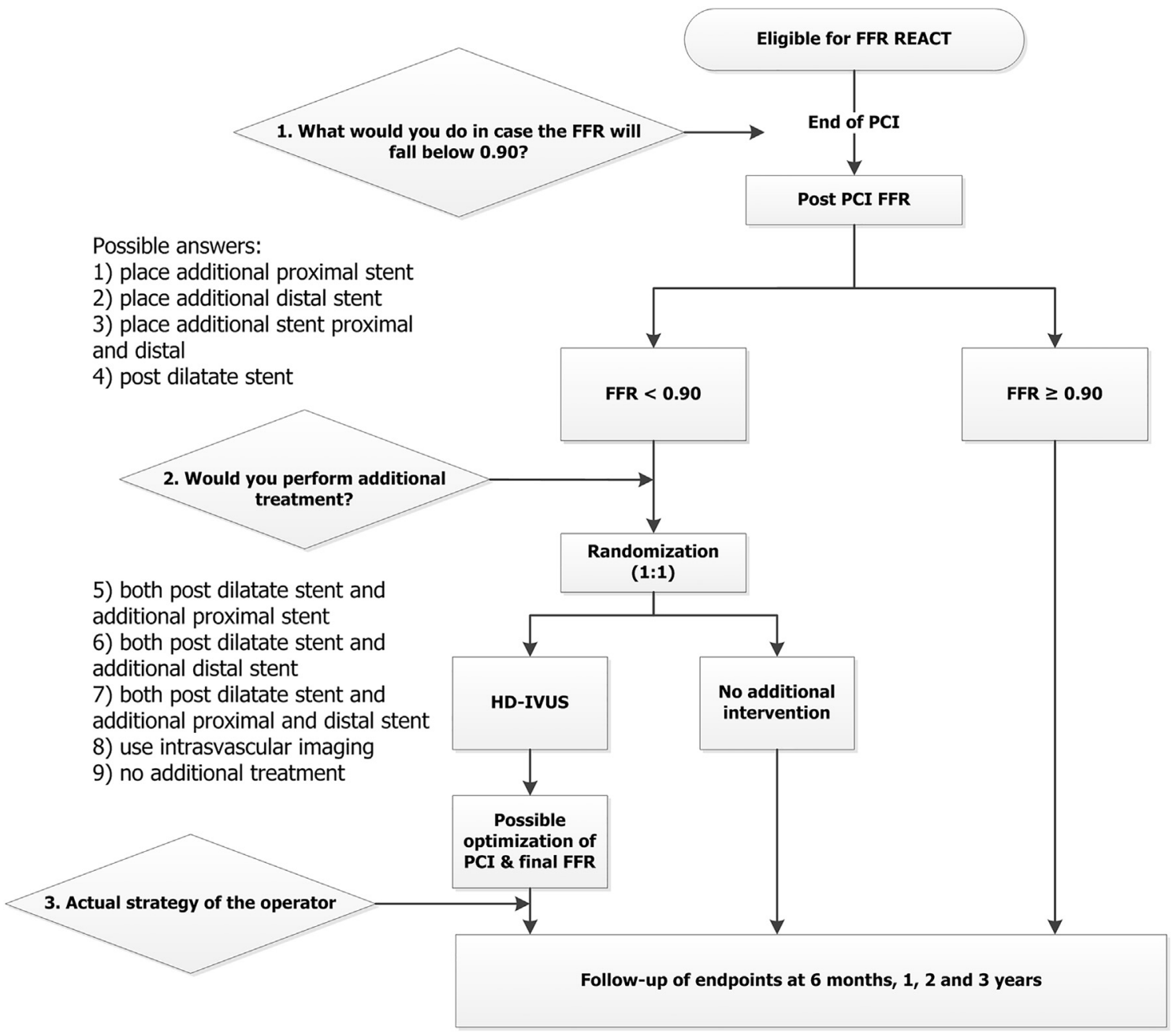

Study flow chart including the operator strategy questionnaire. The first two rhombuses contain the operator questions. The third rhombus is not asked but filled in according to the additional intervention. Nine possible answers are provided. FFR is fractional flow reserve, HD-IVUS is high definition intravascular ultrasound and $\mathrm{PCl}$ is percutaneous coronary intervention.

about post procedural FFR and subsequent treatment arm allocation. More specifically, no information will be provided on the measured vessel, post PCI FFR value and potential randomization allocation in the procedure report or discharge letter. Event adjudication at the set time points of 6 months, 1, 2 and 3 years will be performed by an independent critical event committee, not aware of the patients' specific FFR values and/or randomization allocation. Patients will be unblinded at the last follow-up moment (3 years).

Investigational products. Post PCI FFR will be assessed using the Navvus ${ }^{\circledR}$ monorail microcatheter (ACIST Medical Systems, Inc., Eden Prairie, MN) advanced over the previously used coronary guidewire. This monorail microcatheter precludes the need to advance a separate pressure wire along the treatment segment which will simplify and speed-up post PCI FFR measurements. $^{12}$

Additional imaging in the intervention group will be performed using the multi frequency (40-60 MHz) $\operatorname{Kodama}^{\circledR}$ HD-IVUS catheter (ACIST Medical Systems, Inc., Eden Prairie, MN). Both devices are CE marked and are currently used in regular clinical practice.

\section{Study procedures}

Routine care. Procedures will be performed according to standard clinical practice: angiography guided PCI and stenting with the use of periprocedural imaging, (either IVUS or OCT) and/or pre-procedural functional assessment (either iFR or FFR) left at the discretion of the operator. ${ }^{13}$ Angiographic success was defined as residual stenosis $<30 \%$ by visual analysis in the presence of TIMI 3 grade flow. Procedural success will be identified as 
Table I. In- and exclusion criteria.

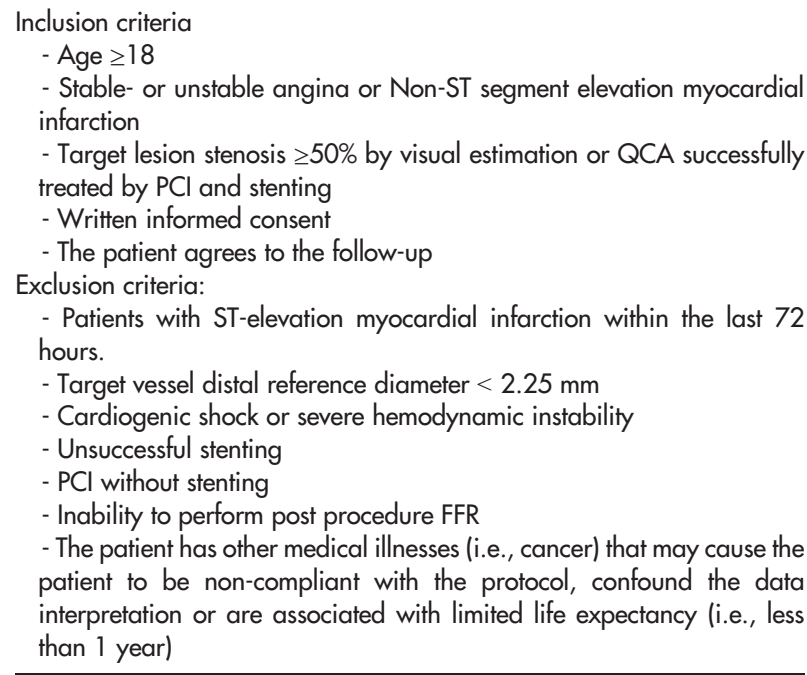

QCA is quantitative coronary angiography, FFR is fractional flow reserve, $\mathrm{PCI}$ is percutaneous coronary intervention.

angiographic success in the absence of periprocedural MI. Dual antiplatelet therapy (including aspirin and a P2Y12 inhibitor) will be prescribed for at least 6 months to all patients consisting of clopidogrel in case of stable angina, or prasugrel/ticagrelor for at least 12 months in case of an acute coronary syndrome. ${ }^{14}$

Study measurements and interventions, if applicable, will only be performed after confirmation of angiographic success of the PCI and after administration of intracoronary nitrates.

\section{Post procedural indices: $\mathrm{Pd} / \mathrm{Pa}$ and FFR}

$\mathrm{Pd} / \mathrm{Pa}$ is defined as a ratio, where $\mathrm{Pd}$ is the distal coronary pressure derived from the tip of the Navvus ${ }^{\circledR}$ catheter and $\mathrm{Pa}$ stands for proximal coronary pressure (measured at the tip of the guiding system). The two values are recorded simultaneously during resting conditions. FFR is defined as mean distal coronary artery pressure divided by mean aortic pressure during maximum hyperemia achieved by continuous intravenous infusion of adenosine at a rate of $140 \mu \mathrm{g} / \mathrm{kg} / \mathrm{min}$ through an antecubital vein.

Both indices will be measured approximately $20 \mathrm{~mm}$ from the most distal stent edge. A pullback will be performed to obtain pressure gradients on the distal and proximal stent edges. Drift will be checked at the end of each pullback. Measurements with a drift value above 0.02 will be repeated a second time. ${ }^{15}$ All vessels with a drift value above 0.05 during the second attempt will be excluded from the study. All pressure tracing will be stored in a dedicated database for off-line analyses. All tracings will be analyzed for ventricularization, dampening and drift by our academic corelab.
Table II. Primary and secondary endpoints of the FFR-REACT trial.

Primary endpoints

Target vessel failure*

Secondary endpoints

In hospital

- Procedural characteristics e.g. contrast medium usage, no. of stents, total stent length and procedural time

- Major access site bleeding

- Periprocedural MI

- Acute kidney injury

- Periprocedural complications

- Change in post-procedural FFR after optimization therapy

- Change in post-procedural Pd/Pa and FFR after optimization therapy

- Correlation of the IVUS parameters and proximal VS stent VS distal FFR drop in categories of 0.05 .

- Correlation of FFR segmental drop and minimum luminal area on IVUS and 3D QCA

- Correlation of $\mathrm{Pd} / \mathrm{Pa}$ and FFR, both dependent and independent of IVUS findings

- Operators $\mathrm{PCl}$ strategy change dependent on the information received from either FFR or IVUS

6 months and longer follow-up

- The individual components of the primary endpoint (cardiac death, target vessel MI, target vessel revascularization)

- All-cause mortality

- Cardiovascular mortality

- Prehospitalization for heart failure

- Target lesion revascularization

- Any coronary revascularization

- Non-fatal myocardial infarction

- Stent thrombosis

- Periprocedural MI

- Stroke

- Kidney injury

- Correlation of $\mathrm{Pd} / \mathrm{Pa}, \mathrm{FFR}$ and the primary endpoints components

*A composite of cardiac death, target-vessel myocardial infarction and clinically driven TVR at 1 year. Ml is myocardial infarction, FFR is fractional flow reserve, QCA is quantitative coronary angiography, $\mathrm{PCl}$ is percutaneous coronary intervention and IVUS is intravascular ultrasound.

\section{Intravascular ultrasound (Intervention Group)}

IVUS-directed FFR optimization will be guided by an automated pullback with a 40-60 MHz HD-IVUS catheter at a speed of $2.5 \mathrm{~mm} / \mathrm{sec}(24$ frames $/ \mathrm{mm}$ ) starting approximately $20 \mathrm{~mm}$ distal from the most distal stent edge. Images will be analyzed online in order to identify potential reasons for the low post-procedural FFR. Treatment of potential anomalies will be performed through a guidance protocol initiated in order to standardize potential further treatment (Table III) and will be based on the patient's characteristics, angiographic anatomy, distal and interval $\mathrm{Pd} / \mathrm{Pa}$ and FFR and luminal IVUS dimensions. Final resting $\mathrm{Pd} / \mathrm{Pa}$ and FFR will be measured at the end of the procedure if additional treatment was performed along with an IVUS pullback assessing the final treatment result.

All IVUS pullbacks will be analyzed offline using QCUCMS (Leiden University MC, LKEB, Division of Image Processing, version 4.69). Offline analysis will be performed by three independent IVUS experts within our academic corelab. All IVUS pullback will be divided in 
Table III. stepwise protocol after high definition IVUS

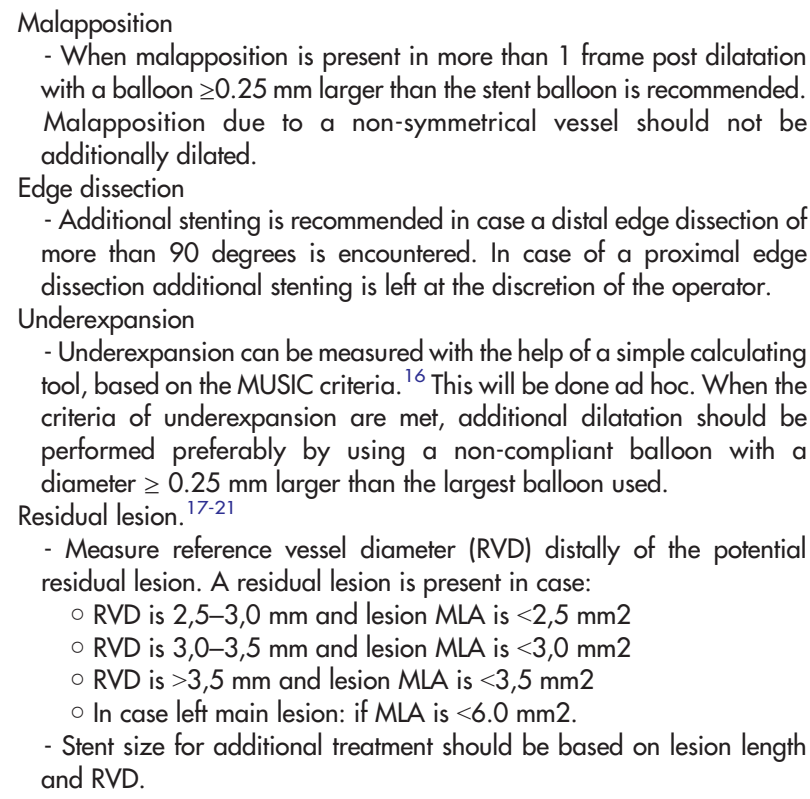

To ensure a homogenous treatment approach post IVUS imaging of the treated segment the following guidelines have been designed.

4 segments, a distal segment, in stent, in segment (stent \pm $5 \mathrm{~mm}$ ) and a proximal segment. The luminal dimensions for all segments will be separately analyzed, including, but not limited to, minimal lumen area, minimal lumen diameter, minimal stent area, mean lumen diameter, mean lumen diameter and maximum plaque burden. Additionally, malapposition, stent edge dissections, underexpansion and residual lesion will be scored according to Table III.

\section{Conservative treatment (Control Group)}

No further treatment or IVUS assessment will be performed. Procedures will be concluded based on the confirmation of angiographic success according to routine clinical practice.

Operator strategy. Operator strategy will be assessed at 3 time points during the procedure based on the available information at that stage: following angiography, following first post-PCI FFR in patients with a FFR $<0.90$, and following HD-IVUS in subjects who are randomized to the IVUS-directed FFR optimization. In the first question, the operator will be asked what he/she would do in the hypothetical case the FFR would fall below 0.90. Possible answers are: 1) place additional proximal stent 2) place additional distal stent 3) place additional stent proximal and distal 4) post dilatate stent 5) both post dilatate stent and additional proximal stent 6 ) both post dilatate stent and additional distal stent 7) both post dilatate stent and additional proximal and distal stent 8 ) perform intravascular imaging 9) no additional treatment. A similar question will be asked directly after the first FFR measurement in which the answer may be guided by the information provided by the initial post PCI Pd/Pa and FFR (pullback) analyses. The latter two answers will be compared to the actual treatment strategy based on the IVUS pullback (Figure 1). The operator strategy questions were added with the intent to further assess how the use of post PCI FFR and IVUS impact treatment strategies intended to improve PCI results.

\section{Follow-up at 6 months, 1,2 and 3 years follow-up}

All patients will be contacted by letter and/or telephone contact at 6, 24 and 36 months. Before patient contact, survival status will be ascertained by an automated civil registry check. A clinical follow-up with ECG will be scheduled at 12 months. All possible clinical outcomes, including all-cause mortality, cardiac mortality, myocardial infarction (MI), target lesion revascularization (TLR) and TVR, any revascularization, stent thrombosis, stroke and bleeding. Additional information will be retrieved in case of event triggers from local electronic medical records, referring physicians and general practitioner.

\section{Data management and monitoring}

Registry of specific endpoints and other details will be managed through OpenClinica, an electronic, online, case report form (CRF) application. Follow-up contacts will be performed by physicians or study nurses not involved in the index procedure and blinded to the final FFR and assigned treatment arm. All data will be anonymized and handled confidentially. The key to the de-anonymization will be safeguarded by the principal investigator.

Event adjudication will be performed by an independent Clinical Events Committee (CEC) unaware of the post PCI FFR and assigned treatment arm. Specific information in the PCI report on the treatment strategy will be masked when submitting documents to the CEC.

Monitoring will verify that the rights and well-being of the patients are protected, the trial is conducted according to GCP and ISO14155, and that the protocol is followed. The trial specific monitoring program is based on the guidelines for on-site monitoring in relationship to the estimated risk of the study (Erasmus MC version 15 November 2012). According to these guidelines a negligible risk-monitoring program was set up for the trial.

\section{Statistical considerations}

Statistical analysis of endpoints. Categorical variables will be expressed as percentages and counts. Differences in categorical variables between randomly allocated treatment groups will be evaluated by applying chi-square tests or Fisher's exact tests. Continuous variables will be described as mean \pm one standard deviation, or as median and interquartile range, accordingly. ShapiroWilk tests will be applied to evaluate normality of continuous variables. Differences in continuous variables 
between randomly allocated treatment groups will then be evaluated by applying Student's t-tests or MannWhitney tests. Parametric correlations will be assessed using the Pearson correlation coefficient while the Spearman's rank correlation coefficient is used if the correlation is non-parametric.

The operator strategy questionnaire will be evaluated using the McNemar's test.

Differences between the groups, both randomized and non-randomized, will be measured using the log-rank tests to evaluate differences in event-free survival. A univariate Cox proportional hazard regression will be used to quantify the relation between randomly allocated treatment arms and the incidence of clinical outcomes. In order to provide adjusted hazard ratios (HR), a multivariate Cox regression will be used, with adjustment for age, sex and (as far as allowed given the number of endpoint events) other confounders, possibly including stent size, previous coronary artery intervention, previous MI, multivessel disease, a history of CABG, dyslipidemia, hypertension, smoking, diabetes mellitus and renal function. Competing risks are taken into account for the analysis. Both primary and secondary study parameters are depicted in Table II.

All tests will be 2 -tailed, and a p-value of $<0.05$ will be considered statistically significant. The secondary outcomes are hypothesis generating and therefore no adjustment for multiple testing will be made. We will report estimates of population parameters together with their 95\% confidence interval.

Sample size calculation. A recent meta-analysis showed that the incidence of MACE (heterogeneous definitions used) in patients with post PCI FFR $<0.90$ was $21.4 \%$ versus $5 \%$ in patients with post PCI FFR $\geq 0.90{ }^{4}$ The average incidence of MACE in the latter study was $11 \%$. The average incidence of MACE (comprised of cardiac death, any MI and TVR) at 1-year post PCI at the Erasmus MC is $10 \%$. When these data are extrapolated, in the Erasmus MC patients with an FFR $<0.90$ will have an estimated MACE incidence of $19 \%$. The MACE incidence of the patients who will be randomized to optimal care with IVUS is estimated at $7.5 \%$ : the average of the incidence at the EMC and the 5\% that was found in the meta-analysis.

In summary, to determine the sample size we made the following assumptions/choices:

- Incidence of the study endpoint in those randomized to control/standard care: $19 \%$

- Incidence of the study endpoint in those randomized to IVUS-directed stent placement: $7.5 \%$

- type I error, two-sided: 0.05 .

- type II error: 0.2 (i.e. power $80 \%$ )

- Allocation ratio N2/N1 = 1 .

Then a sample size of 272 is required, 136 patients per treatment arm. The sample size should be enlarged by an additional $2-5 \%$ due to possible technical failures, lost to follow-up or unsuitable FFR or IVUS acquisition. Finally 290 patients will be randomized.

Based on results of the FFR-SEARCH registry, $45 \%$ of the patients will have a post-procedural FFR $<0.90 .{ }^{10}$ This implies that a total of approximately 640 patients need to be enrolled in order find 290 patients with a post PCI FFR $<0.90$.

\section{Potential issues of concern}

The use of FFR and IVUS in daily clinical practice has been shown to be safe with a low risk of complications. In the FFR SEARCH study, focusing on the predictive value of post-procedural FFR in almost 1000 patients, no complications due to the microcatheter were observed, while in only 2 patients a severe response to the intravenous adenosine occurred. ${ }^{10}$ In a study by van der Sijde et al in which the risk of periprocedural complications due to the use of IVUS was assessed in 2476 procedures, 12 complications $(0.5 \%)$ occurred. ${ }^{22}$ All of these were selflimiting after retrieval of the imaging catheter and no major adverse events due to the use of IVUS were found. Furthermore, limited evidence is available at the moment on the homogeneity of post PCI FFR values in patients presenting with stable angina as compared to patients with unstable angina or non-ST elevation MI. Additionally, pre PCI FFR assessment using the Navvus microcatheter has proven to significantly overestimated the stenosis severity as compared to pressure wire based FFR measurements. ${ }^{23,24}$ However, this difference was mainly driven by a larger delta in vessels with a small minimal luminal area pre procedure. It is currently unknown if post PCI FFR assessment with the latter two methods will exemplify the same variance and direct extrapolation of the current study to pressure wire based post PCI FFR assessment and optimization is therefore not possible.

The sample size calculation presented the current study is based on a meta-analysis which included a heterogeneous cohort of studies with several outdated registries. ${ }^{4}$ The latter might result in an overestimation of the event rates and thus under-power the study design. Cumulative incidences are expected to diverge between the treatment and control group at longer follow-up if the luminal dimension and FFR can be increased, ensuring a hypothetical under-powered study at one year would be sufficiently powered at two or three years follow-up. ${ }^{4,5,25,26}$

\section{Study status and timeline}

The FFR REACT trial is actively enrolling patients since October 31st, 2017 and has reached the milestone of enrolling $50 \%$ of the target population October 2018. At its current pace the study is expected to complete enrolment Q4 2019.

\section{Summary}

The FFR REACT study is an investigator initiated prospective, single-center randomized controlled trial 
conducted at the Erasmus Medical Center designed to assess if FFR guided PCI optimization directed by IVUS in patients with an increased risk for MACE (post-PCI FFR below 0.90) will decrease target vessel failure at 1 year. Inclusion started in October 2017 and enrolment is expected to be complete in Q4 2019.

\section{Public disclosure and publication policy}

Findings of the study will be submitted for publication in a peer-reviewed international cardiology journal. Publication of the data will remain in the hands of the principal investigator and steering committee. The Erasmus MC received an unrestricted institutional grant from ACIST Medical Systems, Inc.

\section{References}

1. Cameron A, Kemp Jr HG, Fisher LD, et al. Left main coronary artery stenosis: angiographic determination. Circulationfive] 1983;68(3):484-9.

2. Girasis C, Onuma Y, Schuurbiers JC, et al. Validity and variability in visual assessment of stenosis severity in phantom bifurcation lesions: a survey in experts during the fifth meeting of the European Bifurcation Club. Catheter Cardiovasc Interv 2012;79(3):361-8.

3. Tonino PA, De Bruyne B, Pijls NH, et al. Fractional flow reserve versus angiography for guiding percutaneous coronary intervention. N Engl J Med 2009;360(3):213-24.

4. Wolfrum M, Fahrni G, de Maria GL, et al. Impact of impaired fractional flow reserve after coronary interventions on outcomes: a systematic review and meta-analysis. BMC Cardiovasc Disord 2016;16(1):177.

5. Rimac G, Fearon WF, De Bruyne B, et al. Clinical value of postpercutaneous coronary intervention fractional flow reserve value: $A$ systematic review and meta-analysis. Am Heart J 2017;183:1-9.

6. Tonino PA, Johnson NP. Why Is Fractional Flow Reserve After Percutaneous Coronary Intervention Not Always 1.0? JACC Cardiovasc Interv 2016;9(10):1032-5.

7. Zandvoort van LC, Masdjedi K, Witberg K, et al. Explanation of postprocedural fractional flow reserve below $0.85 \mathrm{~A}$ comprehensive ultrasound analysis of the FFR SEARCH Registry. Circ Cardivasc Interv 2019;12(2):213-24.

8. Ahn J-M, Kang S-J, Yoon S-H, et al. Meta-analysis of outcomes after intravascular ultrasound-guided versus angiographyguided drug-eluting stent implantation in 26,503 patients enrolled in three randomized trials and 14 observational Studies. Am J Cardiol 2014;113(8):1338-47.

9. Zhang J, Gao X, Kan J, et al. Intravascular ultrasound-guided versus angiography-guided implantation of drug-eluting stent in all-comers: The ULTIMATE trial. J Am Coll Cardiol 2018;72(24): 3126-37, https://doi.org/10.1016/j.jacc.2018.09.013.

10. Bommel van R, Masdjedi K, Diletti R, et al. Routine fractional flow reserve measurement after percutaneous coronary intervention the FFR-SEARCH Study. Circ Cardivasc Interv 2019;12(5), e007428.

11. Cutlip DE, Windecker S, Mehran R, et al. Clinical end points in coronary stent trials: a case for standardized definitions. Circulation 2007;1 15(17):2344-51.

12. Diletti R, Van Mieghem NM, Valgimigli $M$, et al. Rapid exchange ultra-thin microcatheter using fibre-optic sensing technology for measurement of intracoronary fractional flow reserve. Eurolntervention 2015;1 1(4):428-32.

13. Authors/Task Force $m$, Windecker $S$, Kolh $P$, et al. ESC/EACTS Guidelines on myocardial revascularization: The Task Force on Myocardial Revascularization of the European Society of Cardiology (ESC) and the European Association for CardioThoracic Surgery (EACTS)Developed with the special contribution of the European Association of Percutaneous Cardiovascular Interventions (EAPCI). Eur Heart J 2014;2014.

14. Neumann F-J, Sousa-Uva M, Ahlsson A, et al. ESC/EACTS Guidelines on myocardial revascularization. Eur Heart J 2018;2018:ehy394-ehy.

15. Cook Christopher M, Ahmad Y, Shun-Shin Matthew J, et al. Quantification of the effect of pressure wire drift on the diagnostic performance of fractional flow reserve, instantaneous wave-free ratio, and whole-cycle Pd/Pa. Circ Cardiovasc Interv 2016;9(4), e002988.

16. de Jaegere $P$, Mudra $H$, Figulla $H$, et al. Intravascular ultrasoundguided optimized stent deployment. Immediate and 6 months clinical and angiographic results from the Multicenter Ultrasound Stenting in Coronaries Study (MUSIC Study). Eur Heart J 1998;19 (8): 1214-23.

17. Waksman R, Legutko J, Singh J, et al. FIRST: Fractional Flow Reserve and Intravascular Ultrasound Relationship Study. J Am Coll Cardiol 2013;61(9):917-23.

18. Ben-Dor I, Torguson R, Deksissa T, et al. Intravascular ultrasound lumen area parameters for assessment of physiological ischemia by fractional flow reserve in intermediate coronary artery stenosis. Cardiovasc Revasc Med 2012;13(3):177-82.

19. Ben-Dor I, Torguson R, Gaglia Jr MA, et al. Correlation between fractional flow reserve and intravascular ultrasound lumen area in intermediate coronary artery stenosis. Eurolntervention 2011;7 (2):225-33.

20. Mallidi J, Atreya AR, Cook J, et al. Long-term outcomes following fractional flow reserve-guided treatment of angiographically ambiguous left main coronary artery disease: A meta-analysis of prospective cohort studies. Catheter Cardiovasc Interv 2015;86 (1):12-8

21. de la Torre Hernandez JM, Baz Alonso JA, Gomez Hospital JA, et al. Clinical impact of intravascular ultrasound guidance in drug-eluting stent implantation for unprotected left main coronary disease: pooled analysis at the patient-level of 4 registries. JACC Cardiovasc Interv 2014;7(3):244-54.

22. van der Sijde JN, Karanasos A, van Ditzhuijzen NS, et al. Safety of optical coherence tomography in daily practice: a comparison with intravascular ultrasound. Eur Heart J Cardiovasc Imaging 2017;18(4):467-74.

23. Pouillot C, Fournier S, Glasenapp J, et al. Pressure wire versus microcatheter for FFR measurement: a head-to-head comparison. Eurolntervention 2018;13(15):e1850-e6.

24. Wijntjens GW, van de Hoef TP, Kraak RP, et al. The IMPACT Study (Influence of Sensor-Equipped Microcatheters on Coronary Hemodynamics and the Accuracy of Physiological Indices of Functional Stenosis Severity). Circ Cardiovasc Interv 2016;9 (12).

25. Elgendy IY, Mahmoud AN, Elgendy AY, et al. Outcomes with intravascular ultrasound-guided stent implantation: a metaanalysis of randomized trials in the era of drug-eluting stents. Circ Cardiovasc Interv 2016;9(4), e003700.

26. Witzenbichler B, Maehara A, Weisz G, et al. Relationship between intravascular ultrasound guidance and clinical outcomes after drug-eluting stents: the Assessment of Dual Antiplatelet Therapy With Drug-Eluting Stents (ADAPT-DES) Study. Circulation 2014;129(4):463-70. 